\title{
THUẬT TOÁN NỘI SUY VÀ VẼ MẶT CẮT ĐỊA HÌNH TRÊN MÔ HİNH TIN
}

\author{
NGUYẼN QUANG KHÁNH \\ Trường Đại học Mỏ - Địa chất
}

\section{Tóm tắt:}

Nội suy mặt cắt là việc làm thường xuyên của công tác tư vấn khảo sát địa hình, mô hình TIN được dùng để mô phỏng bề mặt địa hình và là dữ liệu cơ sở cho hầu hết các ứng dụng nội suy và phân tích địa hình trên máy tính. Bài báo này trình bày cách thức thành lập và lưu trữ dư liệu mô hình số địa hình TIN, từ đó xây dụ̂ng thuật toán và chương trình máy tính nội suy mặt cắt địa hình, vẽ và hiển thị dữ liệu mặt cắt địa hình dạng số.

\section{1. Đặt vấn đề}

Mô hình số địa hình thường được dùng để biểu diễn bề mặt địa hình trên máy tính. Trong công tác khảo sát thiết kế các công trình xây dựng, giao thông, thủy lợi... người ta thường sử dụng mô hình số địa hình dạng lưới tam giác không đều ký hiệu là TIN. Mô hình này được lưu trữ trên máy tính bao gồm một tập các tam giác liền kề và tập các điểm đo địa hình [1].

Một trong những ứng dụng của mô hình số địa hình là cho phép chúng ta tạo các mặt cắt địa hình trên mô hình số. Trước đây chúng ta thường làm những công việc này trên những phần mềm nhập ngoại, bài báo này sẽ đưa ra thuật toán và xây dựng một chương trình máy tính để thực nghiệm và khả năng áp dụng thuật toán vào thực tế.

\section{Cấu trúc dữ liệu và thuật toán thành lập mô hình TIN}

\subsection{Dũr liệu mô hình TIN}

Cấu trúc dữ liệu mô hình số địa hình được thực hiện theo dạng topo gồm danh sách các điểm, danh sách các tam giác và danh sách các cạnh như thể hiện trong Hình 1 . Việc lưu trữ dữ liệu mô hình TIN có thể được thực hiện như mô hình lưu trữ topo (Hình 1) hoặc lưu trữ dưới dạng cơ sở dữ liệu quan hệ gồm 3 bảng DIEM, bảng TAMGIAC và bảng CANH như Hình 2.

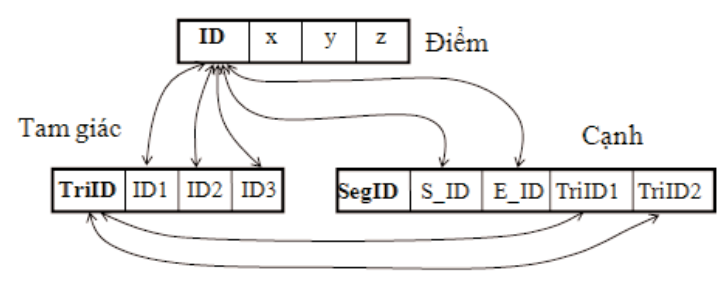

Hình 1: Dũ̃ liệu dạng cấu trúc của mô hình TIN

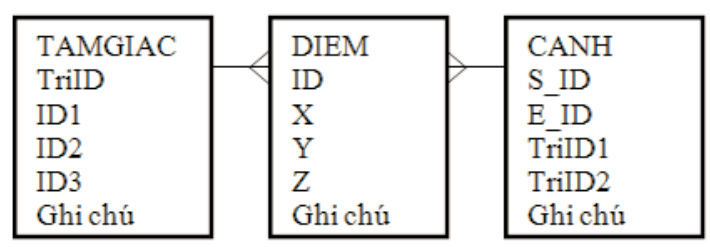

Hình 2: Dũr liệu dạng bảng của mô hình TIN

Ngày nhận bài: 13/11/2016, ngày chuyển phản biện: 15/11/2016, ngày chấp nhận phản biện: 16/12/2016, ngày chấp nhận đăng: 19/12/2016 


\subsection{Thành lập mô hình TIN theo thuật toán Tăng tiến}

Thuật toán Tăng tiến [2] thực hiện việc tam giác hóa bề mặt địa hình theo nguyên lý: Khi thêm một điểm vào mô hình TIN thì sẽ chia tam giác chứa điểm đó thành nhiều tam giác nhỏ và thực hiện kiểm tra các tam giác đó để thỏa mãn điều kiện là các tam giác tối ưu. Do các điểm thêm vào nằm ngẫu nhiên trên toàn mặt phẳng nên việc kiểm tra các tam giác này được tiến hành trên tất cả các tam giác hiện có trên mô hình TIN và thời gian tính toán trong trường hợp xấu nhất là $\mathrm{O}\left(\mathrm{n}^{2}\right)$, trường hợp tốt nhất là $\mathrm{O}(n \log n)$ phụ thuộc vào sự phân bố của các điểm đặc trưng địa hình. Từ $n$ điểm trong mặt phẳng, thuật toán Tăng tiến sẽ tạo ra một lưới tam giác thỏa mãn tiêu chuẩn Delaunay và được dùng để mô phỏng địa hình trên máy tính (mô hình TIN).

\section{Bài toán nội suy độ cao trên mô hình TIN}

Nội suy độ cao trên mô hình TIN là dựa vào tọa độ, độ cao của các điểm lân cận cùng với một hàm toán học thích hợp để nội suy độ cao của điểm cần xác định và thường sử dụng phương pháp nội suy tuyến tính [1]. Nội dung của phương pháp là sử dụng một hàm tuyến tính, xác định độ cao của điểm nằm bên trong một tam giác thông qua độ cao đã biết tại ba đỉnh của tam giác. (Xem hình 3)

Cho ba điểm đã biết tọa độ $\mathrm{M}_{1}\left(\mathrm{x}_{1}, \mathrm{y}_{1}, \mathrm{z}_{1}\right), \mathrm{M}_{2}\left(\mathrm{x}_{2}, \mathrm{y}_{2}, \mathrm{z}_{2}\right)$ và $\mathrm{M}_{3}\left(\mathrm{x}_{3}, \mathrm{y}_{3}, \mathrm{z}_{3}\right)$, cần nội suy độ cao $z$ của một điểm $P(x, y, z)$ nằm trong tam giác $\mathrm{M}_{1} \mathrm{M}_{2} \mathrm{M}_{3}$. Do điểm $\mathrm{P}$ nằm trên mặt phẳng của tam giác $\mathrm{M}_{1} \mathrm{M}_{2} \mathrm{M}_{3}$ nên ba véc tơ $\overrightarrow{M_{1} P}, \overrightarrow{M_{1} M_{2}}$ và $\overrightarrow{M_{1} M_{3}}$ đồng phẳng, nghĩa là tích hỗn hợp của ba véc tơ bằng 0 :

$$
\left(\overrightarrow{M_{1} P}, \overrightarrow{M_{1} M_{2}}, \overrightarrow{M_{1} M_{3}}\right)=0
$$

Từ đây suy ra công thức tính độ cao điểm $\mathrm{P}$ :

$$
\mathrm{z}=\mathrm{z}_{1}-\frac{\left(\mathrm{x}-\mathrm{x}_{1}\right)\left(\mathrm{y}_{21} \cdot \mathrm{z}_{31}-\mathrm{y}_{31} \cdot \mathrm{z}_{21}\right)+\left(\mathrm{y}-\mathrm{y}_{1}\right)\left(\mathrm{z}_{21} \cdot \mathrm{x}_{31}-\mathrm{z}_{31} \cdot \mathrm{x}_{21}\right)}{\mathrm{x}_{21} \cdot \mathrm{y}_{31}-\mathrm{y}_{21} \cdot \mathrm{x}_{31}}
$$

trong đó:

$$
\left\{\begin{array}{lll}
\mathrm{x}_{21}=\mathrm{x}_{2}-\mathrm{x}_{1} & ; & \mathrm{x}_{31}=\mathrm{x}_{3}-\mathrm{x}_{1} \\
\mathrm{y}_{21}=\mathrm{y}_{2}-\mathrm{y}_{1} & ; & \mathrm{y}_{31}=\mathrm{y}_{3}-\mathrm{y}_{1} \\
\mathrm{z}_{21}=\mathrm{z}_{2}-\mathrm{z}_{1} & ; & \mathrm{z}_{31}=\mathrm{z}_{3}-\mathrm{z}_{1}
\end{array}\right.
$$

\section{Bài toán nội suy mặt cắt trên mô hình TIN}

Bài toán tổng quát như sau: Mặt cắt $\mathrm{AB}$ cắt mô hình $\mathrm{TIN}$ tại $\mathrm{m}_{\mathrm{i}}$ điểm $(i=1 \div /)$ như vậy cần xác định tọa độ $/$ điểm $m_{i}$ là giao của các cạnh $j k(j=1 \div n, k=1 \div n)$ với mặt cắt $A B$ và nội suy độ cao tại các điểm đó. (Xem hình 4)

Ví dụ như khảo sát một mạng lưới tam giác TIN gồm 11 điểm đánh số từ $1 \div 11$ và 12 tam giác như Hình 3 , cần lập mặt cắt địa hình theo hướng $A B$ với $A^{\top}, m_{i}^{\top} B^{\top}$ là đỉnh mặt cắt tương ứng với các điểm $A, m_{i}$ và $B$. Do các điểm từ $1 \div 11$ là các điểm đặc trưng của địa 


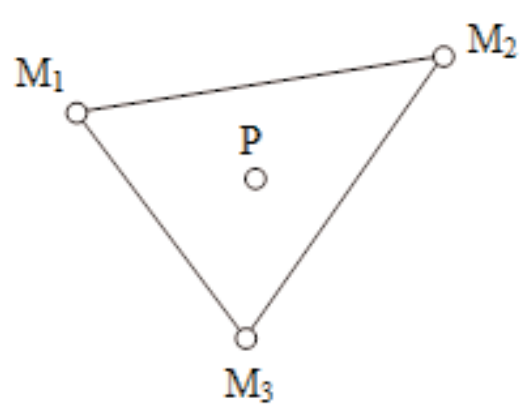

Hình 3 : Nội suy độ cao điểm $P$

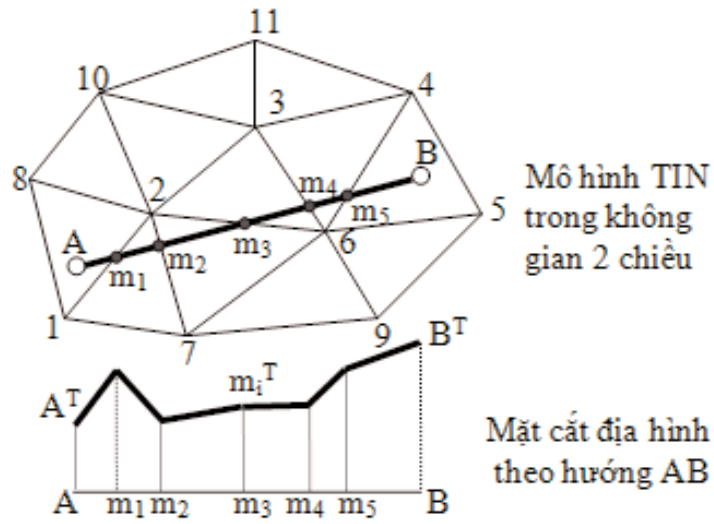

Hình 4 : Mặt cắt địa hình trên mô hình TIN

hình, nên các cạnh của tam giác tạo bởi các điểm đó là các đường đặc trưng địa hình. Vì vậy, giao điểm $m_{i}$ của mặt cắt $A B$ với các cạnh của tam giác cũng chính là các điểm đặc trưng địa hình. (Xem hình 5)

Trước tiên, cần xác định $\mathrm{m}_{\mathrm{i}}(i=1 \div 5)$ là giao điểm của mặt cắt $A B$ với các cạnh của tam giác trên mô hình. Mỗi giao điểm này chính là nghiệm của hệ hai phương trình, một của đoạn thẳng $A B$ và một của cạnh tam giác mà đoạn $A B$ cắt qua. Giả sử cần xác định điểm $\mathrm{m}_{1}(\mathrm{x}, \mathrm{y})$. Ta có phương trình tham số của đoạn thẳng $A B$ và đoạn thẳng là cạnh của tam giác đi qua hai điểm $\left(\mathrm{x}_{1}, \mathrm{y}_{1}\right)$ và $\left(\mathrm{x}_{2}, \mathrm{y}_{2}\right)$ có dạng như công thức $(4)$ :

$$
\left\{\begin{array} { l } 
{ x = ( 1 - t ) x _ { A } + t x _ { B } } \\
{ y = ( 1 - t ) y _ { A } + t y _ { B } }
\end{array} \quad \left\{\begin{array}{l}
x=\left(1-t^{\prime}\right) x_{1}+t^{\prime} x_{2} \\
y=\left(1-t^{\prime}\right) y_{1}+t^{\prime} y_{2}
\end{array} \text { với } t, t^{\prime} \in[0,1]\right.\right.
$$

Giải hệ phương trình được:

$$
\begin{aligned}
& t=\frac{\left(y_{1}-y_{A}\right) \cdot\left(x_{2}-x_{1}\right)-\left(x_{1}-x_{A}\right) \cdot\left(y_{2}-y_{1}\right)}{\left(x_{2}-x_{1}\right) \cdot\left(y_{B}-y_{A}\right)-\left(y_{2}-y_{1}\right) \cdot\left(x_{B}-x_{A}\right)} \\
& t^{\prime}=\frac{\left(y_{1}-y_{A}\right) \cdot\left(x_{B}-x_{A}\right)-\left(x_{1}-x_{A}\right) \cdot\left(y_{B}-y_{A}\right)}{\left(x_{2}-x_{1}\right) \cdot\left(y_{B}-y_{A}\right)-\left(y_{2}-y_{1}\right) \cdot\left(x_{B}-x_{A}\right)}
\end{aligned}
$$

Thay $\mathrm{t}$ hoặc $\mathrm{t}^{\prime}$ vào (4) xác định được giá trị tọa độ $\mathrm{x}, \mathrm{y}$. Nếu $\mathrm{t} \in[0,1]$ thì giá trị $\mathrm{x}, \mathrm{y}$ chính là tọa độ của điểm $m_{1}$. Cứ lần lượt như vậy ta sẽ xác định được tọa độ của tất cả các điểm giao khác $\mathrm{m}_{\mathrm{i}}(i=2 \div 5)$.

Tiếp theo, chúng ta cần xác định độ cao của các điểm giao $m_{\mathrm{i}}(i=1 \div 5)$. Khảo sát trường hợp xác định độ cao của điểm $\mathrm{m}_{1}$. Từ Hình 4 ta thấy, điểm $\mathrm{m}_{1}$ nằm trên cạnh nối giữa hai điểm 1 và 2 . Độ cao của hai điểm 1 và 2 đã biết là $z_{1}$ và $z_{2}$, khoảng cách từ điểm 1 đến điểm $\mathrm{m}_{1}$ là $\mathrm{d}_{1}$, từ điểm 1 đến điểm 2 là $\mathrm{d}_{12}$. Nếu coi độ cao của địa hình biến đổi tuyến tính từ điểm 1 đến điểm 2 thì độ cao của điểm $\mathrm{m}_{1}$, tính theo độ cao điểm 1 , sẽ tỷ lệ thuận với 
khoảng cách tới điểm 1 và tỷ lệ nghịch với khoảng cách giữa hai điểm 1 và 2 . Công thức tính độ cao điểm $\mathrm{m}_{1}$ như sau:

$$
\mathrm{z}=\mathrm{z}_{1}+\frac{\mathrm{d}_{1}}{\mathrm{~d}_{12}} \cdot\left(\mathrm{z}_{2}-\mathrm{z}_{1}\right)
$$

trong đó: $\mathrm{d}_{1}=\sqrt{\left(\mathrm{x}-\mathrm{x}_{1}\right)^{2}+\left(\mathrm{y}-\mathrm{y}_{1}\right)^{2}}$ và $\mathrm{d}_{12}=\sqrt{\left(\mathrm{x}_{1}-\mathrm{x}_{2}\right)^{2}+\left(\mathrm{y}_{1}-\mathrm{y}_{2}\right)^{2}}$

Cuối cùng là việc xác định độ cao của hai điểm đầu và điểm cuối của mặt cắt $A B$. Độ cao của $A$ và $B$ được xác định theo công thức (2).

Thuật toán nội suy mặt cắt địa hình:

1. Input: tập các tam giác thuộc TIN, tọa độ các đỉnh các tam giác và tọa độ điểm đầu $A$ và điểm cuối $B$ của mặt cắt.

2. Nội suy độ cao của điểm $A$ và $B$ theo công thức (2).

3. Xác định giao điểm $\mathrm{m}_{\mathrm{i}}$ của mặt cắt $\mathrm{AB}$ với các cạnh của tam giác trên mô hình số địa hình theo công thức (4).

4. Nội suy độ cao của các điểm giao $\mathrm{m}_{\mathrm{i}}$ theo công thức (5).

5. Kết thúc.

Thuật toán vẽ mặt cắt địa hình:

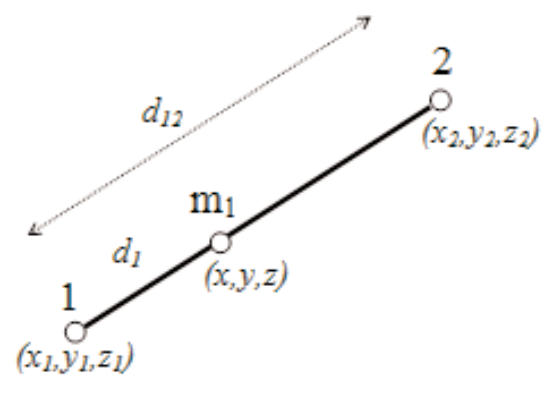

Hình 5 : Nội suy độ cao của điểm $\mathrm{m}_{1}$

1. Input: tập các điểm tọa độ-độ cao trên mặt cắt địa hình $\left(\mathrm{A}, \mathrm{B}, \mathrm{m}_{\mathrm{i}}\right)$.

2. Tính khoảng cách giữa các điểm trên tuyến mặt cắt $\left(\mathrm{S}_{\mathrm{Am} 1}, \mathrm{~S}_{\mathrm{m} 1 \mathrm{~m} 2}, \ldots \mathrm{S}_{\mathrm{mnB}}\right)$ theo công thức (6).

3. Xác định tọa độ điểm $A$ (có thể chọn bất kỳ trên bản vẽ), tính tọa độ các điểm $m_{i}$ và $B$ theo hướng ngang sử dụng khoảng cách tính trong bước 2 .

4. Tại các điểm $A, m_{i}$ và $B$ vẽ đường thẳng theo hướng đứng với khoảng cách bằng độ cao $z_{A}, z_{m i}, z_{B}$. Xác định được tọa độ các điểm $A^{\top}, m_{i}^{\top}$ và $B^{\top}$.

5. Vẽ đường thẳng nối các điểm $\mathrm{A}^{\top}, \mathrm{m}_{\mathrm{i}}^{\top}$ và $\mathrm{B}^{\top}$ và trình bày mặt cắt địa hình theo tỷ lệ.

6. Kết thúc.

\section{Xây dựng chương trình xác định mặt cắt địa hình và thực nghiệm}

\subsection{Xây dựng chương trình}

Trong [1] đã xây dựng một phần mềm đồ họa để tương tác biểu diễn, biên tập, lưu trữ bề mặt địa hình dạng TIN. Giao diện chương trình được thiết kế theo dạng chuẩn của Windows bao gồm: menu điều khiển, phím tắt, các thanh công cụ (tools bar), thanh trạng thái (status bar), thanh cuốn (scroll bar) và vùng hiển thị tương tác (Hình 6). 
Trên cơ sở dữ liệu bề mặt địa hình, mô đun nội suy và vẽ mặt cắt địa hình được thành lập bằng ngôn ngữ lập trình VB6 trên nền đồ họa mã nguồn mở VeCAD và thực hiện theo 3 bước như sau: (Xem hình 7)

\subsection{Thực nghiệm}

Trên cơ sở thuật toán vẽ mặt cắt địa hình, đề tài này đã thành lập mô đun vẽ mặt cắt tự động trên mô hình số địa hình với các bước thao tác như sau: H).

1. Nhập số liệu điểm đo (thực nghiệm với số liệu có 605 điểm với tọa độ $\mathrm{x}, \mathrm{y}$ và độ cao

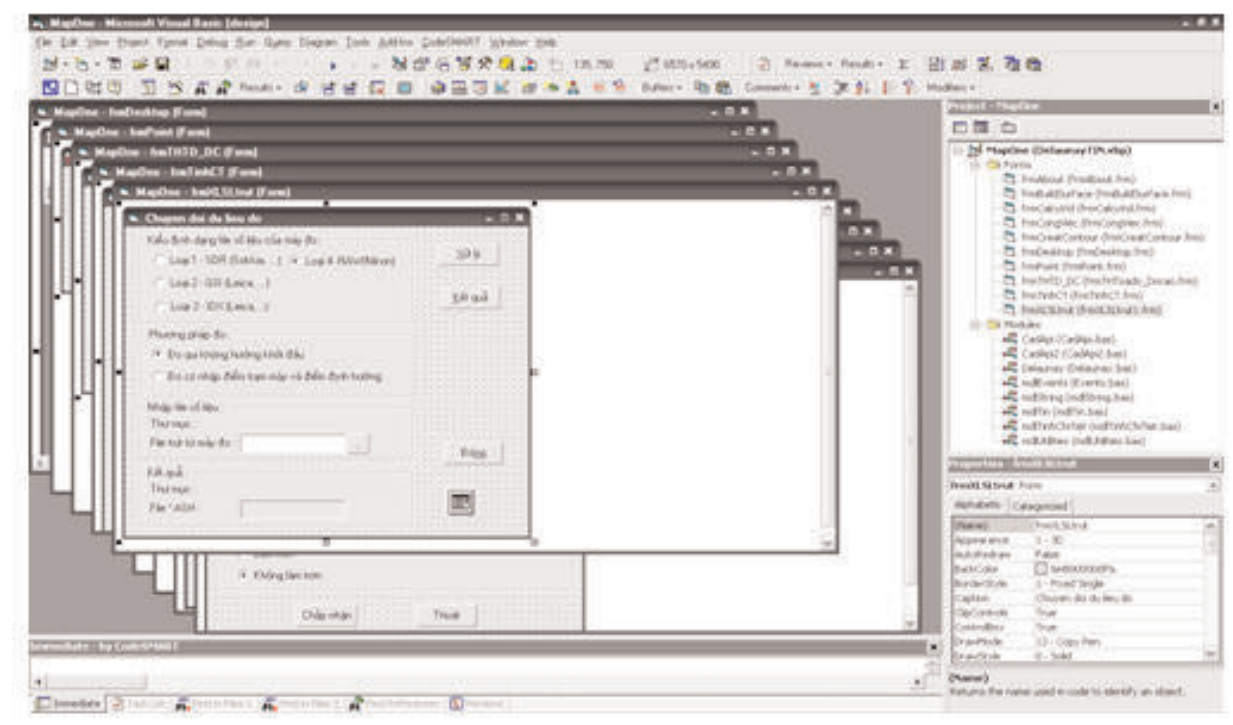

Hình 6: Thiết kế giao diện và lập trình trình trên Visual Basic 6

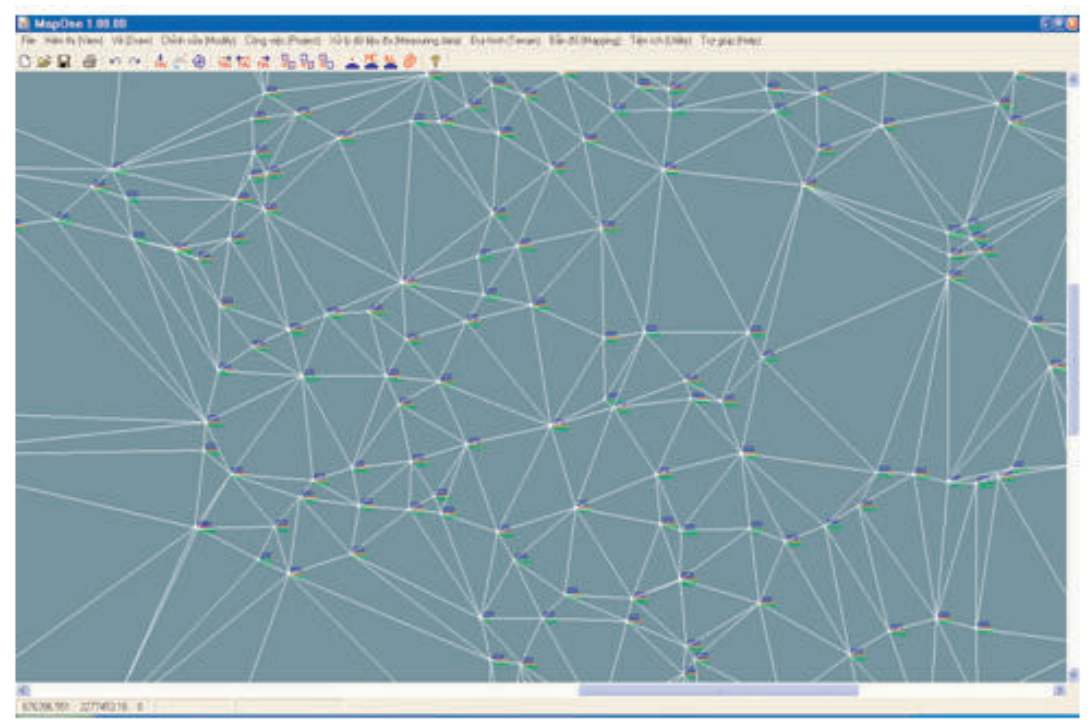

Hình 7: Giao diện chương trình 
2. Xây dựng mô hình TIN (với 605 điểm thực nghiệm, số tam giác tạo được trong mô hình TIN là 1191 tam giác và số cạnh tạo được là 1779 cạnh).

3. Vẽ mặt cắt: nhấn chuột vào điểm đầu mặt cắt và điểm cuối mặt cắt. Chương trình sẽ tự động xác định giao điểm của mặt cắt với các cạnh của tam giác trên mô hình số địa hình, đồng thời nội suy độ cao tại các vị trí này. Cuối cùng là vẽ mặt cắt ra màn hình hiển thị (Hình 8).

Việc nội suy và vẽ mặt cắt đã được so sánh với chương trình SoftDeck 8.0 (Hình 9) và cho kết quả tương đương.

\section{Kết luận}

- Mô hình số địa hình dạng TIN là một dạng dữ liệu nền tảng, cho phép chúng ta khai thác, sử dụng cho nhiều mục đích khác nhau. Việc xây dựng các thuật toán và các mô dun ứng dụng đã tự động hóa được hầu hết các công đoạn của thiết kế khảo sát, thi công. Nội suy tự động mặt cắt địa hình và vẽ lại trên nền bản đồ số cho kết quả nhanh chóng, chính xác, tránh được nhầm lẫn.

- Kết quả thực nghiệm thuật toán nội suy và vẽ mặt cắt địa hình đã đảm bảo tính chính xác và có thể dùng để phát triển thành công cụ tiện ích trong các phần mềm GIS và bản đồ số. $\mathrm{O}$

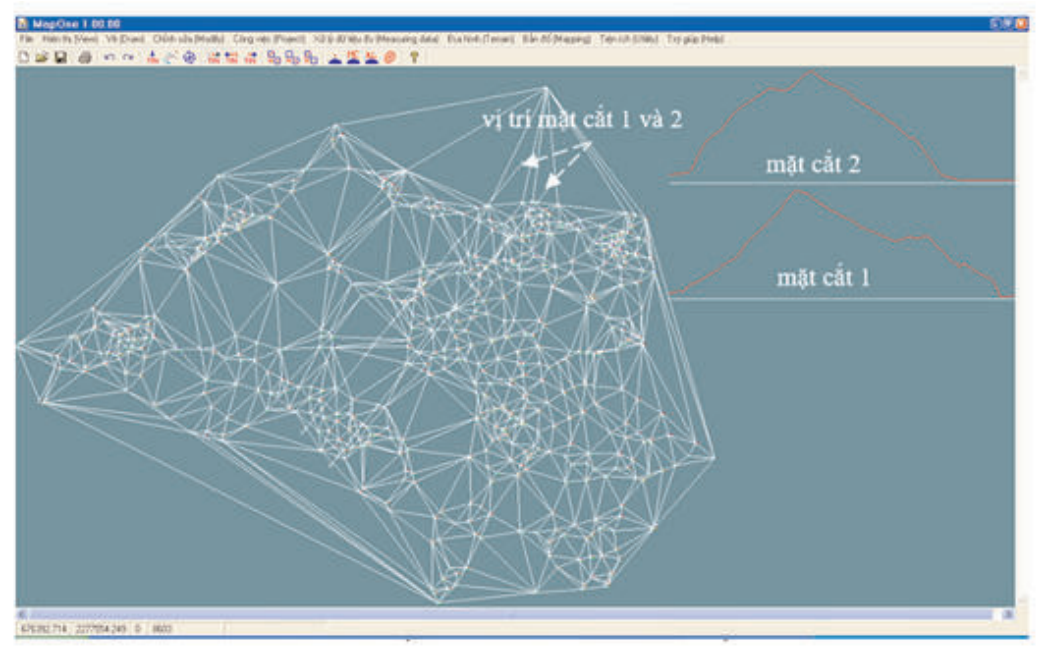

Hình 8: Vẽ mặt cắt địa hình (minh họa cho quy trình thao tác trên phần mềm)

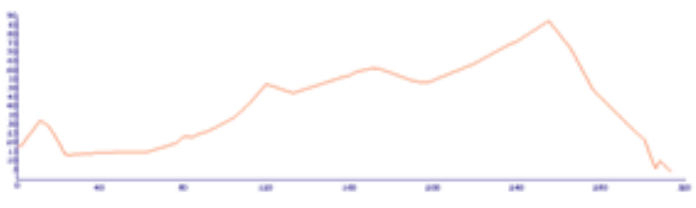

(a) Mặt cắt xác định được theo mục 4

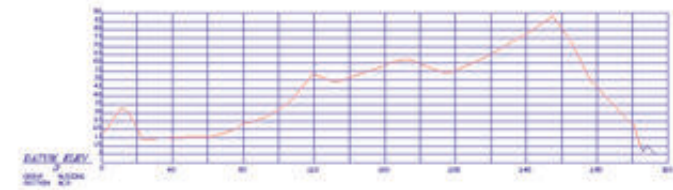

(b) Mặt cắt vẽ bằng SoftDesk 8.0

Hình 9: Hình ảnh mặt cắt địa hình (minh họa kết quả so sánh với phần mềm khác) 


\section{Tài liệu tham khảo}

[1]. Nguyễn Quang Khánh, Nghiên cứu thuật toán và xây dựng hệ phần mềm quản lý khai thác cơ sở dũ liệu địa hình, Luận án Tiến sĩ kỹ thuật, 2011, 133tr.

[2]. Mark de Berg, Marc van Kreveld, Computational Geometry Algorithms and Application, 2000.

[3]. Ron Singh, David Artman, David W. Taylor, Dave Brinton, Basic surveying Theory and Practice, 2000, Ninth Annual Seminar. $O$

\section{Summary}

\section{The algorithm for interpolating and drawing the terrain sections from TIN model}

Nguyen Quang Khanh, University of Mining and Geology

Interpolation terrain section is regular work of technical consultant, TIN models are used to simulate the surface terrain and it is the data for most of the terrain analysis applications on the computer. This paper presents how to establish and store digital terrain data as model TIN, from which to build algorithms and computer programs for interpolating the terrain sections, drawing and displaying it. $O$

\section{MỘT PHƯƠNG PHÁP TÍNH THỂ TÍCH......}

(Tiếp theo trang 16)

\section{Summary}

\section{One method to caculate volume of 3D object using TIN}

Tran Thuy Duong, Ngo Thi Lien, University of Mining and Geology

Le Quang Hung, Resource Enviroment and Materials Technology Joinstock Company

When studying 3D objects, one of the important properties of practical significance is to calculate volume. Calculating of the volume can be used for a lot of applications in many brands such as: in designing drainage systems for roads; in dredging the waterway traffic; in digging and filling engineering constructions; in mining in the design, inventory for management and exploitation of minerals; in calculating the volume of civil engineering,... Recently there have been some reseaches which gave different solutions to solve this problem by TIN model. However, by studying the methods mentioned above, these solutions are complex in calculating and are not optimized, the data collection process must be followed by some definite conditions. This paper gives a method for calculating the volume in the general case for 3D objects with any shape. According to this method, the caculated process becomes simple, the organization of field work is flexible, multiple ways and not complicated. $\bigcirc$ 